\title{
Nucleation-Elongation Polymerization under Imbalanced Stoichiometry Supporting Information
}

Dahui Zhao and Jeffrey S. Moore*

Departments of Chemistry and Material Science \& Engineering University of Illinois, Urbana, Illinois 61801

\section{Characterization of Polymerization Products}
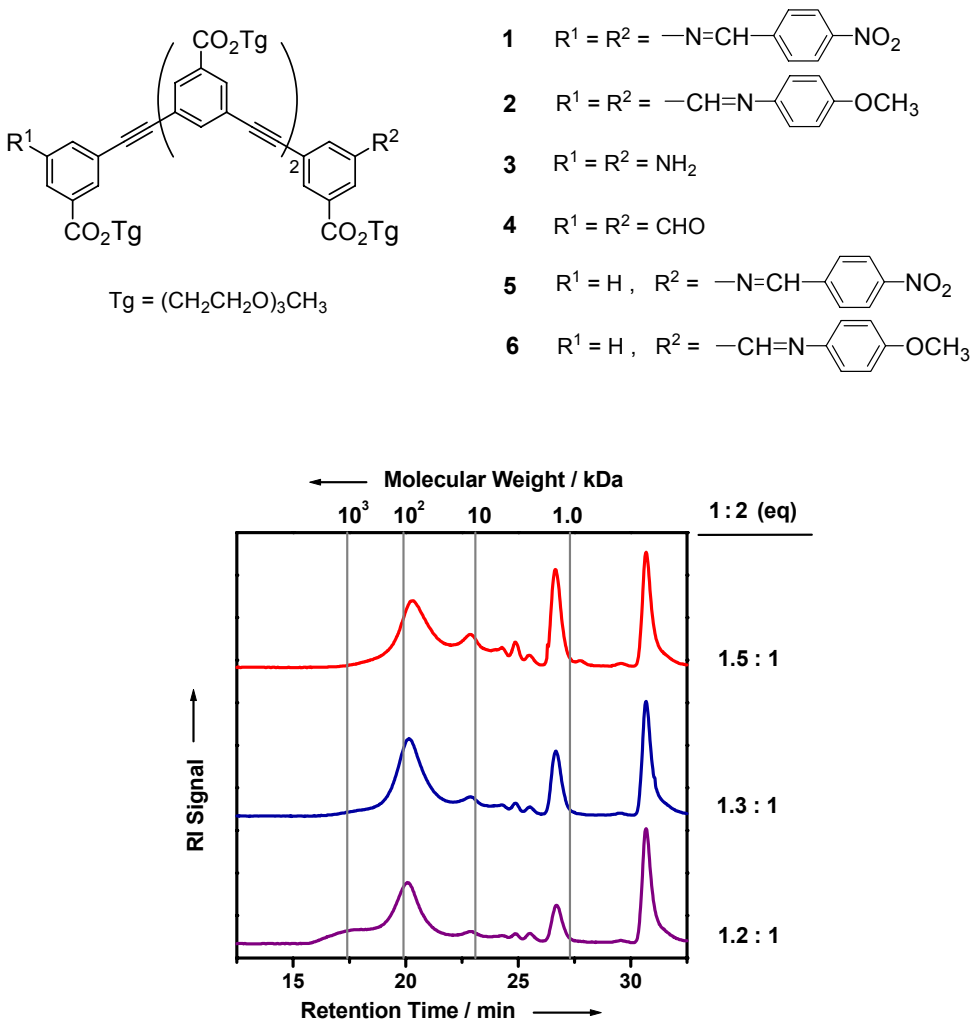

Figure S1. SEC traces of polymerization products equilibrated in the presence of $0.5 \mathrm{mM}$ oxalic acid for $14 \mathrm{~d}$ at $\mathrm{rt}$ in $\mathrm{CH}_{3} \mathrm{CN}$, with the initial molar ratios of $\mathbf{1}$ and $\mathbf{2}$ as specified with each trace. The reactions were quenched with $\mathrm{Et}_{3} \mathrm{~N}$ prior to SEC analysis (eluted by THF). The initial concentration of 2 was kept constant at $5 \mathrm{mM}$ in all reactions. Polymerizations at high molar ratios with $\mathbf{1}$ in excess were not possible due to the limited solubility of $\mathbf{1}$ in acetonitrile.

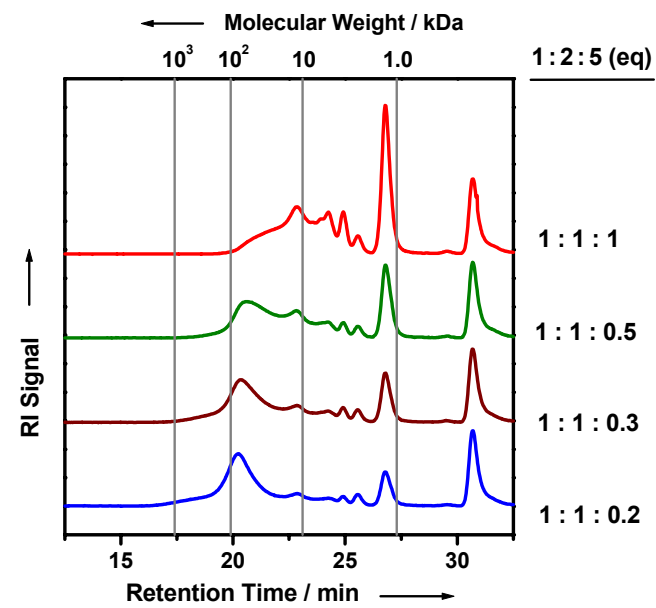

Figure S2. SEC traces of polymerization products equilibrated in the presence of $0.5 \mathrm{mM}$ oxalic acid for $14 \mathrm{~d}$ at $\mathrm{rt}$ in $\mathrm{CH}_{3} \mathrm{CN}$, starting with a mixture of $\mathbf{1 , 2}$ and $\mathbf{5}$ at molar ratios as specified with each trace. The reactions were quenched with $\mathrm{Et}_{3} \mathrm{~N}$ prior to SEC analysis. In each polymerization, concentrations of $\mathbf{1}$ and $\mathbf{2}$ remained constant at $5 \mathrm{mM}$. 


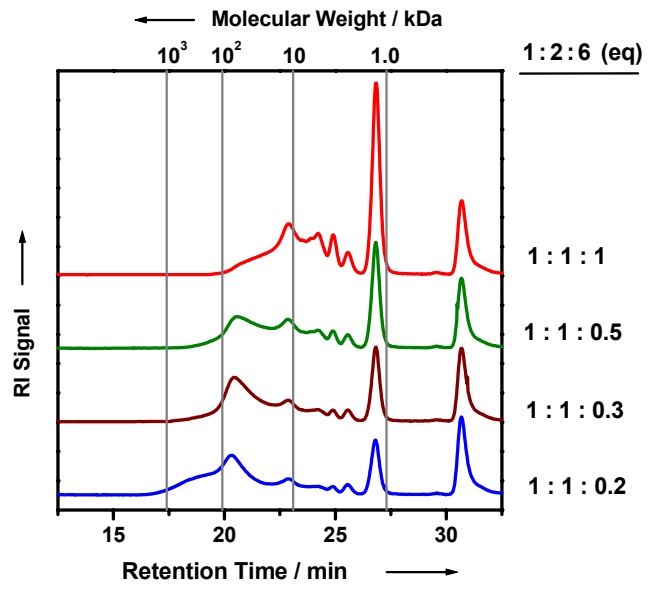

Figure S3. SEC traces of polymerization products equilibrated in the presence of $0.5 \mathrm{mM}$ oxalic acid for $14 \mathrm{~d}$ at $\mathrm{rt}$ in $\mathrm{CH}_{3} \mathrm{CN}$, starting with a mixture of $\mathbf{1 , 2}$ and $\mathbf{6}$ at molar ratios as specified with each trace. The reactions were quenched with $\mathrm{Et}_{3} \mathrm{~N}$ prior to SEC analysis. In each polymerization, concentrations of $\mathbf{1}$ and $\mathbf{2}$ remained constant at $5 \mathrm{mM}$.

\section{Model}

\section{Scheme S1}

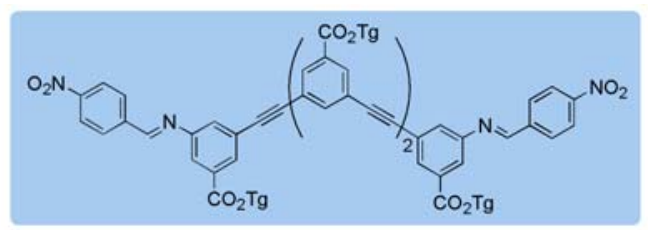

A

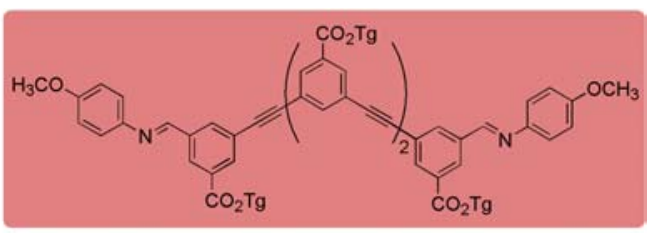

B

$1 \mathrm{H}^{+}$(cat.)

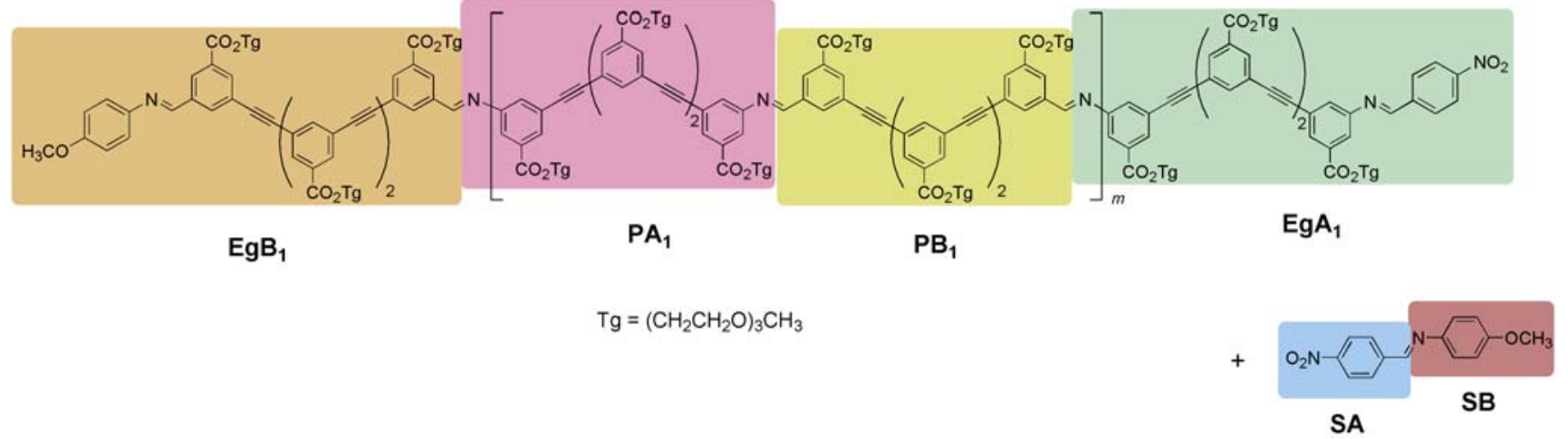

In the mechanistic scheme of the model:

$$
\left.\begin{array}{l}
\mathrm{A}+\mathrm{B} \stackrel{K_{1}}{\rightleftharpoons} \mathrm{EgA}+\mathrm{EgB} \\
\mathrm{A}+\mathrm{EgB} \stackrel{K_{2}}{\rightleftharpoons} \mathrm{EgA}+\mathrm{PB} \\
\mathrm{B}+\mathrm{EgA} \stackrel{K_{2}}{\rightleftharpoons} \mathrm{EgB}+\mathrm{PA}
\end{array}\right\}
$$

A and B stand for the starter sequences as shown in Scheme S1, while other species represent combinations of structural fragments: $\mathrm{EgA}=\mathrm{EgA} 1+\mathrm{SA}, \mathrm{EgB}=\mathrm{EgB} 1+\mathrm{SB}, \mathrm{PA}=\mathrm{PA} 1+2 \mathrm{SA}, \mathrm{PB}=\mathrm{PB} 1+2 \mathrm{SB}$. It should be noted that $\mathrm{EgA}, \mathrm{EgB}, \mathrm{PA}$ 
and PB are "conceptual molecules" and cannot exist independently. Nevertheless, since the total number of "conceptual molecules" is consistent with the total number of real molecules in the metathesis polymerization (including the byproduct molecule), mathematically it is viable to use Eq 1 to analyze the thermodynamic characteristics of the real polymerization system, provided that appropriate equilibrium constant is selected for each step.

\section{Characterizations of 1-6}

Starter sequences 1-6 are synthesized as previously reported. ${ }^{1,2}$ Additional characterization data are provided here on the particular samples that are used in the current studies.

Bisimine 1. ${ }^{1} \mathrm{H}$ NMR $\left(500 \mathrm{MHz}, \mathrm{CDCl}_{3}\right) \delta 8.64(\mathrm{~s}, 2 \mathrm{H}), 8.37(\mathrm{~m}, 4 \mathrm{H}), 8.21(\mathrm{~m}, 4 \mathrm{H}), 8.16(\mathrm{t}, \mathrm{J}=1.5 \mathrm{~Hz}, 2 \mathrm{H}), 8.13(\mathrm{~m}$, 4H), $7.93(\mathrm{t}, \mathrm{J}=1.4 \mathrm{~Hz}, 2 \mathrm{H}), 7.91(\mathrm{t}, \mathrm{J}=1.6 \mathrm{~Hz}, 2 \mathrm{H}), 7.63(\mathrm{t}, \mathrm{J}=1.8 \mathrm{~Hz}, 2 \mathrm{H}), 4.64(\mathrm{~m}, 8 \mathrm{H}), 3.88(\mathrm{~m}, 8 \mathrm{H}), 3.74(\mathrm{~m}, 8 \mathrm{H}), 3.69$ (m,

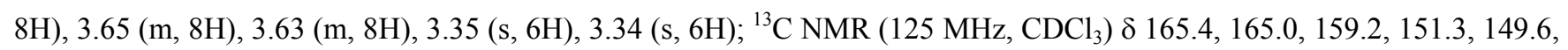
141.0, 138.4, 132.8, 132.7, 131.8, 131.1, 131.0, 129.7, 128.4, 124.1, 123.7, 123.6, 122.2, 89.4, 89.0, 88.7, 71.9, 70.60, 70.57, 69.1, 69.0, 64.5, 59.0; LRMS (FAB): $[\mathrm{M}+\mathrm{Na}]^{+}$calcd 1457.5, found 1457.1; HRMS (FAB): $[\mathrm{M}+\mathrm{Na}]^{+}$calcd for $\mathrm{C}_{76} \mathrm{H}_{82} \mathrm{~N}_{4} \mathrm{O}_{24} \mathrm{Na}$, 1457.5217, found 1457.5233; Anal. Calcd for $\mathrm{C}_{76} \mathrm{H}_{82} \mathrm{~N}_{4} \mathrm{O}_{24}$ (1435.48): C, 63.59; H, 5.76; N, 3.90; Found: C, $63.50 ; \mathrm{H}, 5.82 ; \mathrm{N}, 3.95$.

Bisimine 2. ${ }^{1} \mathrm{H}$ NMR $\left(500 \mathrm{MHz}, \mathrm{CDCl}_{3}\right) \delta 8.55(\mathrm{~s}, 2 \mathrm{H}), 8.49(\mathrm{t}, \mathrm{J}=1.6 \mathrm{~Hz}, 2 \mathrm{H}), 8.33(\mathrm{t}, \mathrm{J}=1.5 \mathrm{~Hz}, 2 \mathrm{H}), 8.30(\mathrm{t}, \mathrm{J}=1.4 \mathrm{~Hz}$, 2H), $8.22(\mathrm{~m}, 4 \mathrm{H}), 7.92(\mathrm{t}, \mathrm{J}=1.5 \mathrm{~Hz}, 2 \mathrm{H}), 7.29(\mathrm{~m}, 4 \mathrm{H}), 6.96(\mathrm{~m}, 4 \mathrm{H}), 4.64(\mathrm{~m}, 8 \mathrm{H}), 3.88(\mathrm{~m}, 8 \mathrm{H}), 3.86(\mathrm{~s}, 3 \mathrm{H}), 3.74(\mathrm{~m}, 8 \mathrm{H})$ $3.70(\mathrm{~m}, 8 \mathrm{H}), 3.66(\mathrm{~m}, 8 \mathrm{H}), 3.53(\mathrm{~m}, 8 \mathrm{H}), 3.35(\mathrm{~s}, 6 \mathrm{H}), 3.34(\mathrm{~s}, 6 \mathrm{H}) ;{ }^{13} \mathrm{C} \mathrm{NMR}\left(125 \mathrm{MHz}, \mathrm{CDCl}_{3}\right) \delta 165.4,165.1,158.8$, 155.6, 144.0, 138.4, 137.2, 135.0, 134.7, 132.8, 132.7, 131.2, 131.0, 130.0, 123.7, 123.6, 122.4, 114.5, 89.5, 89.0, 88.8, 72.2, 71.9, 70.7, 70.65, 70.60, 69.1, 69.0, 64.6, 64.5, 59.03, 59.02, 55.5; LRMS (FAB): $[\mathrm{M}+\mathrm{H}]^{+}$calcd 1405.6, found 1405.9; HRMS (FAB): $[\mathrm{M}+\mathrm{H}]^{+}$calcd for $\mathrm{C}_{78} \mathrm{H}_{89} \mathrm{~N}_{2} \mathrm{O}_{22}, 1405.5907$, found 1405.5920; Anal. Calcd for $\mathrm{C}_{78} \mathrm{H}_{88} \mathrm{~N}_{2} \mathrm{O}_{22}$ (1405.53): C, 66.65; H, 6.31; N, 1.99; Found: C, 66.32; H, 6.23; N, 2.13.

Bisamine 3. ${ }^{1} \mathrm{H}$ NMR (500 MHz, $\left.\mathrm{CDCl}_{3}\right) \delta 8.17(\mathrm{~d}, \mathrm{~J}=1.6 \mathrm{~Hz}, 4 \mathrm{H}), 7.87$ (t, J=1.6 Hz, 2H), $7.62(\mathrm{t}, \mathrm{J}=1.4 \mathrm{~Hz}, 2 \mathrm{H}), 7.37$ (dd, J=2.3, 1.5 Hz, 2H), 7.03 (dd, J=2.3, $1.3 \mathrm{~Hz}, 2 \mathrm{H}), 4.62(\mathrm{~m}, 4 \mathrm{H}), 4.47$ (m, 4H), 3.89 (br, 4H), 3.87 (m, 4H), 3.73 (m, 8H), $3.70(\mathrm{~m}, 8 \mathrm{H}), 3.66(\mathrm{~m}, 8 \mathrm{H}), 3.64(\mathrm{~m}, 8 \mathrm{H}), 3.36(\mathrm{~s}, 6 \mathrm{H}), 3.35(\mathrm{~s}, 6 \mathrm{H}) ;{ }^{13} \mathrm{C} \mathrm{NMR}\left(125 \mathrm{MHz}, \mathrm{CDCl}_{3}\right) \delta 165.1,164.5,146.6$ 138.4, 132.7, 132.4, 131.5, 130.9, 124.0, 123.6, 123.5, 123.1, 121.6, 116.4, 90.4, 89.0, 87.5, 71.9, 70.7, 70.6, 69.1, 69.0, 64.5, 64.2, 59.0; LRMS (FAB): $[\mathrm{M}+\mathrm{H}]^{+}$calcd 1169.5, found 1169.9; HRMS (FAB): $[\mathrm{M}+\mathrm{H}]^{+}$calcd for $\mathrm{C}_{62} \mathrm{H}_{77} \mathrm{~N}_{2} \mathrm{O}_{20}, 1169.5070$, found 1169.5077; Anal. Calcd for $\mathrm{C}_{62} \mathrm{H}_{76} \mathrm{~N}_{2} \mathrm{O}_{20}$ (1169.27): C, 63.69; H, 6.55; N, 2.40; Found: C, 63.98; H, 6.50; N, 2.51 .

Bisaldehyde 4. ${ }^{1} \mathrm{H}$ NMR (500 MHz, $\left.\mathrm{CDCl}_{3}\right) \delta 10.09$ (s, 2H), 8.53 (t, J=1.6 Hz, 2H), $8.46(\mathrm{t}, \mathrm{J}=1.7 \mathrm{~Hz}, 2 \mathrm{H}), 8.24(\mathrm{t}, \mathrm{J}=1.6$ Hz, 2H), $8.22(\mathrm{~d}, \mathrm{~J}=1.4 \mathrm{~Hz}, 4 \mathrm{H}), 7.92(\mathrm{t}, \mathrm{J}=1.6 \mathrm{~Hz}, 2 \mathrm{H}), 4.58(\mathrm{~m}, 2 \mathrm{H}), 4.54(\mathrm{~m}, 4 \mathrm{H}), 3.88(\mathrm{~m}, 4 \mathrm{H}), 3.73(\mathrm{~m}, 4 \mathrm{H}), 3.70(\mathrm{~m}$, 4H), $3.66(\mathrm{~m}, 4 \mathrm{H}), 3.54(\mathrm{~m}, 4 \mathrm{H}), 3.36(\mathrm{~s}, 6 \mathrm{H}), 3.35(\mathrm{~s}, 6 \mathrm{H}),{ }^{13} \mathrm{C} \mathrm{NMR}\left(125 \mathrm{MHz}, \mathrm{CDCl}_{3}\right) \delta$ 190.6, 165.0, 164.8, 138.5, 137.9, 136.8, 136.0, 133.0, 132.9, 131.7, 131.1, 130.8, 124.4, 123.6, 123.3, 89.8, 89.0, 88.5, 71.9, 70.6, 70.6, 69.1, 69.0, 64.8, 64.6, 
59.0; LRMS (FAB): $[\mathrm{M}+\mathrm{Na}]^{+}$calcd 1217.5, found 1217.5; HRMS (FAB): $[\mathrm{M}+\mathrm{H}]^{+}$calcd for $\mathrm{C}_{64} \mathrm{H}_{75} \mathrm{O}_{22}, 1195.4750$, found 1195.4768; Anal. Calcd for $\mathrm{C}_{64} \mathrm{H}_{76} \mathrm{O}_{22}$ (1195.26): C, 64.31; H, 6.24; Found: C, 63.91; H, 6.14.

Monoimine 5. ${ }^{1} \mathrm{H}$ NMR $\left(500 \mathrm{MHz}, \mathrm{CDCl}_{3}\right) \delta 8.64(\mathrm{~s}, 1 \mathrm{H}), 8.37(\mathrm{~m}, 2 \mathrm{H}), 8.24(\mathrm{~m}, 1 \mathrm{H}), 8.21(\mathrm{~m}, 4 \mathrm{H}), 8.16(\mathrm{~m}, 1 \mathrm{H}), 8.13$ (m, 2H), $8.16(\mathrm{~m}, 1 \mathrm{H}), 7.93(\mathrm{~m}, 1 \mathrm{H}), 7.90(\mathrm{~m}, 2 \mathrm{H}), 7.73(\mathrm{~m}, 1 \mathrm{H}), 7.62(\mathrm{~m}, 1 \mathrm{H}), 7.47(\mathrm{t}, \mathrm{J}=7.8 \mathrm{~Hz}, 1 \mathrm{H}), 4.53(\mathrm{~m}, 8 \mathrm{H}), 3.87(\mathrm{~m}$ 8H), $3.74(\mathrm{~m}, 8 \mathrm{H}), 3.69(\mathrm{~m}, 8 \mathrm{H}), 3.66(\mathrm{~m}, 8 \mathrm{H}), 3.53(\mathrm{~m}, 8 \mathrm{H}), 3.36(\mathrm{~s}, 3 \mathrm{H}), 3.35(\mathrm{~s}, 9 \mathrm{H}) ;{ }^{13} \mathrm{C} \mathrm{NMR}\left(125 \mathrm{MHz}, \mathrm{CDCl}_{3}\right) \delta$ $165.6,165.3,165.0,164.9,159.2,151.2,149.5,140.9,138.3,135.8,132.8,132.7,132.5,131.7,131.0,130.9,130.5,129.8$, $129.6,128.5,128.3,124.0,123.8,123.6,123.5,123.4,122.9,122.2,89.9,89.4,89.0,88.9,88.7,88.2,71.8,70.6,70.55$, 70.52, 69.1, 69.0, 64.5, 64.3, 58.9; LRMS (FAB): $[\mathrm{M}+\mathrm{H}]^{+}$calcd 1287.5, found 1287.4; HRMS (FAB): $[\mathrm{M}+\mathrm{H}]^{+}$calcd for $\mathrm{C}_{69} \mathrm{H}_{79} \mathrm{~N}_{2} \mathrm{O}_{22}$, 1287.5124, found 1287.5113; Anal. Calcd for $\mathrm{C}_{69} \mathrm{H}_{78} \mathrm{~N}_{2} \mathrm{O}_{22}$ (1287.36): C, 64.38; H, 6.11 N, 2.18; Found: C, 64.20; H, 6.02; N, 2.69.

Monoimine 6. ${ }^{1} \mathrm{H}$ NMR $\left(500 \mathrm{MHz}, \mathrm{CDCl}_{3}\right) \delta 8.55(\mathrm{~s}, 1 \mathrm{H}), 8.49(\mathrm{~m}, 1 \mathrm{H}), 8.33(\mathrm{~m}, 1 \mathrm{H}), 8.30(\mathrm{~m}, 1 \mathrm{H}), 8.24(\mathrm{~m}, 1 \mathrm{H}), 8.21$ (m, 4H), $8.06(\mathrm{~m}, 1 \mathrm{H}), 7.91(\mathrm{~m}, 2 \mathrm{H}), 7.73(\mathrm{~m}, 1 \mathrm{H}), 7.47(\mathrm{t}, \mathrm{J}=7.7 \mathrm{~Hz}, 1 \mathrm{H}), 7.29(\mathrm{~m}, 2 \mathrm{H}), 6.96(\mathrm{~m}, 2 \mathrm{H}), 4.54(\mathrm{~m}, 8 \mathrm{H}), 3.88(\mathrm{~m}$, 8H), $3.85(\mathrm{~s}, 3 \mathrm{H}), 3.74(\mathrm{~m}, 8 \mathrm{H}), 3.69(\mathrm{~m}, 8 \mathrm{H}), 3.66(\mathrm{~m}, 8 \mathrm{H}), 3.53(\mathrm{~m}, 8 \mathrm{H}), 3.36(\mathrm{~s}, 3 \mathrm{H}), 3.35(\mathrm{~s}, 6 \mathrm{H}), 3.34(\mathrm{~s}, 3 \mathrm{H}) ;{ }^{13} \mathrm{C} \mathrm{NMR}$ $\left(125 \mathrm{MHz}, \mathrm{CDCl}_{3}\right) \delta 165.7,165.3,165.1,165.0,158.8,155.6,143.9,138.4,137.2,135.9,135.0,134.6,132.9,132.7,132.5$, $131.2,131.0,130.9,130.5,130.0,129.8,128.6,123.8,123.7,123.6,123.5,123.0,122.4,114.4,89.9,89.4,89.0,88.9,88.7$, 88.2, 71.9, 70.6, 70.59, 70.56, 69.1, 69.08, 69.05, 69.01, 64.5, 64.3, 59.0, 55.4; LRMS (FAB): $[\mathrm{M}+\mathrm{H}]^{+}$calcd 1272.5, found 1272.4; HRMS (FAB): $[\mathrm{M}+\mathrm{H}]^{+}$calcd for $\mathrm{C}_{70} \mathrm{H}_{82} \mathrm{NO}_{21}$, 1272.5379, found 1272.5385; Anal. Calcd for $\mathrm{C}_{70} \mathrm{H}_{81} \mathrm{NO}_{21}$ (1272.39): C, 66.08; H, 6.42; N, 1.10; Found: C, 66.01; H, 6.38; N, 1.31 .

\section{References}

1. Oh, K.; Jeong, K.-S.; Moore, J. S. Nature, 2001, 414, 889-893.

2. Zhao, D.; Moore, J. S. J. Am. Chem. Soc. 2002, 124, 9996-9997. 